\title{
Mind Maps e Mental Models: Comparação Conceitual e Aplicação de Webibliomining para Construção de Núcleos de Pesquisa
}

Felipe dos Santos Braga (Mestrando em Engenharia de Produção - UFF)

E-mail: flpsbraga@gmail.com

\author{
Valdecy Pereira (Doutor em Engenharia de Produção - UFF) \\ E-mail: valdecy.pereira@gmail.com
}

Ruben Huamanchumo Gutierrez (Doutor em Engenharia de Produção - UFRJ)

E-mail: rubenhg3000@gmail.com

\section{Resumo}

Principais Pontos Abordados: Considerando os conceitos de "Mind Maps" e "Mental Models", este artigo apresenta uma revisão bibliográfica comparativa breve dos dois termos, assim como o resultado da utilização de técnicas de webibliomining para construção de núcleos de pesquisa referenciais para ambos. Utilizando esta metodologia, este artigo busca responder às seguintes perguntas de pesquisa: Os termos "Mind Maps" e "Mental Models" são correspondentes ao mesmo conceito? Como os temas têm evoluído no tocante à representação na literatura? Método: Busca de artigos na base de dados Scopus utilizando os termos selecionados para a pesquisa, considerando todo o horizonte temporal no qual os trabalhos foram apresentados. Construção e apresentação de indicadores bibliométricos que possam auxiliar na resposta às perguntas de pesquisa e construção de conjunto inicial de referências bibliográficas para os dois conceitos. Limitações: Este estudo se limitou às publicações indexadas na base de dados Scopus até o mês de novembro de 2018. Originalidade: Utilização prática de técnicas de webibliomining para a comparação entre dois conceitos e construção de núcleos de partida para referência teórica. Conclusões e Resultados: Utilizando como base o referencial teórico levantado, foi possível identificar que os conceitos não tratam do mesmo assunto, mesmo considerando a tradução semelhante para o idioma português. Considerando a diferença entre as significações dos termos, foi possível a utilização de técnicas de webibliomining para a definição de um núcleo inicial de referencial teórico para cada um dos conceitos, contendo 15 artigos para a temática "Mind Maps" e 43 artigos para a temática "Mental Models".

Palavras-Chaves: Mind Maps; Mental Models; Modelos Mentais; Mapas Mentais; Webibliomining; Bibliometria; Webmetria 


\section{Introdução}

Neste capítulo será apresentado um arcabouço teórico breve acerca dos temas-chave desta pesquisa, assim como uma análise comparativa visando identificar as similaridades entre os conceitos, caso existam.

\subsubsection{Visão Geral - Mind Maps}

O conceito de Mapas Mentais (Mind Maps) foi criado por Tony Buzan, em 1970, enquanto o autor buscava entender o funcionamento do cérebro visando a maximização do aproveitamento de funções como o aprendizado, a memória e o pensamento criativo. Por meio de seis passos principais - nos quais é altamente estimulada a utilização de cores e imagens, visando à ativação do lado direito do cérebro - é possível a criação de um “emulador da linha de pensamento humana" que permite a captura de e apresentação de um grande volume de ideias em uma única página. (YOAP, 2006)

Para a construção de um mapa mental, devem ser seguidos os seguintes passos:

a) Identifique a ideia central do Mapa, por meio de palavra ou imagem, no centro da página. As principais preocupações nesta etapa deverão ser a representação fiel do assunto a ser abordado e a forma de apresentação, uma vez que a ideia deverá claramente ser identificada como o conceito estrutural do mapa do qual surgirão ramificações;

Figura 1 - Ideia Central

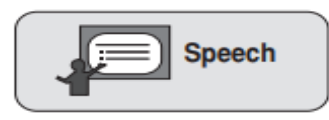

Fonte: Adaptado de YOAP (2006, p. 86)

b) Crie ramificações a partir da ideia central. As ramificações deverão ser apresentadas como linhas curvas que partem da ideia central para seus itens correlatos, preferencialmente afinando-se enquanto se afastam do conceito estrutural. O objetivo desta representação é a verdadeira emulação do modo de pensamento do cérebro humano; 
Figura 2 - Ramificação Principal

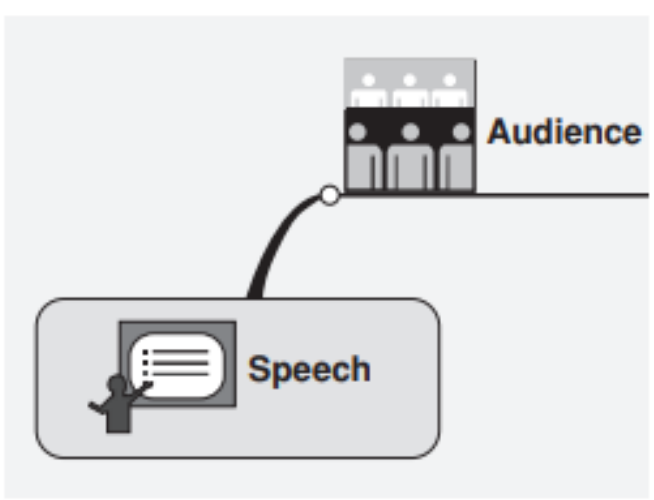

Fonte: Adaptado de YOAP (2006, p. 86)

c) Nomeie a ramificação, preferencialmente com uma única palavra, escrita em letras maiúsculas. Pequenas frases também poderão ser utilizadas para a nomenclatura dos ramos, porém, nomenclaturas superiores a uma palavra irão dificultar a memorização do mapa mental e aumentar o tempo necessário para a análise do diagrama final. Deve-se, portanto, avaliar a relação entre a capacidade de comunicação do mapa e a de análise rápida;

d) Adicione palavras e/ou imagens desencadeadas pela primeira ramificação, representando-as com a utilização de linhas mais finas e fontes menores. Em geral, as mesmas regras utilizadas para a primeira ramificação deverão ser novamente consideradas;

Figura 3 - Ramificações Secundárias

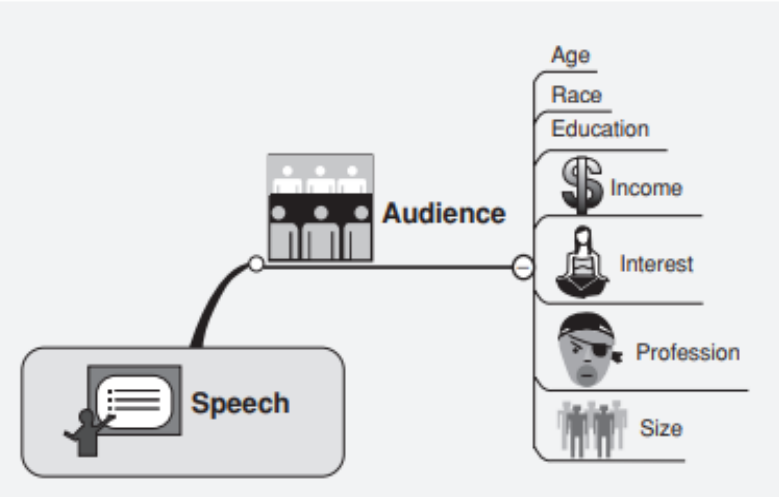

Fonte: Adaptado de YUAP (2006, p. 87)

e) Explicite todas as ideias relacionadas à ramificação principal, seguindo as mesmas regras e direcionamentos dispostos no passo anterior; 
f) Crie novas ramificações a partir da ideia central e percorra novamente todos os passos identificados, até que as ideias para ramificação sejam esgotadas.

Figura 4 - Mapa Mental Completo

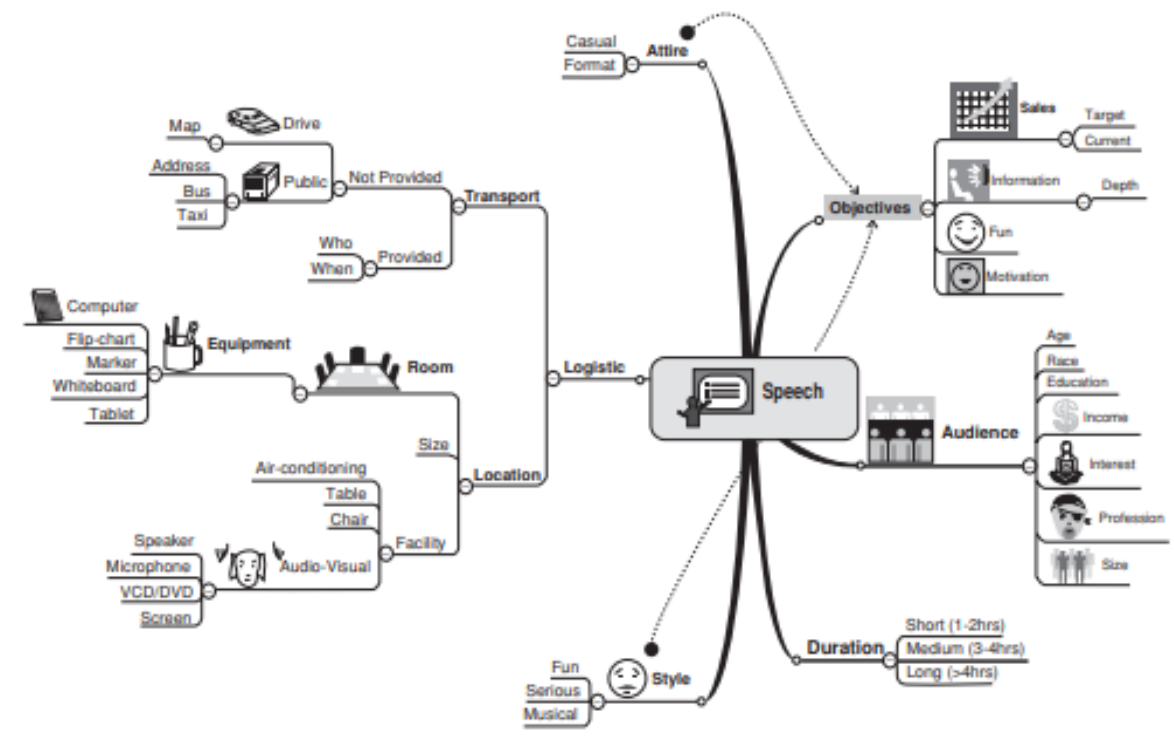

Fonte: Adaptado de YUAP (2006, p. 88)

Conforme pode ser observado, os mapas mentais são ferramentas para a análise de desdobramentos de ideias principais, tendo como concorrentes metodológicos outras metodologias de análise de causas e efeitos e de visualização.

Segundo Eppler (2006), as vantagens da metodologia Mind Maps incluem a facilidade de aplicar e aprender, o encorajamento da criatividade, a concisão da visualização hierárquica e a facilidade de inclusão de novos conceitos e ideias. Esta técnica representa, portanto, um modo no qual o processo de pensamento é representado visualmente pela conexão de conceitos e ideias relacionadas a um problema ou assunto central, de acordo com Somers et al (2014, p. 2 apud Buzan, 1995).

\subsubsection{Visão Geral - Mental Models}

"Um modelo mental é uma representação interna de informações que corresponde analogamente com aquilo que está sendo representado.” (MOREIRA, 1996).

Modelos mentais podem ser - e geralmente o são - representados como blocos cognitivos, que, combinados de diferentes maneiras e formas, podem representar objetos e/ou situações de acordo com a perspectiva de percepção do sujeito. Modelos mentais podem ser, ainda, 
generalizações - ou imagens - que influenciam o modo dos indivíduos entenderem e interagirem com o mundo. (DANIEL 2018, p. 7 apud SENGE, 1990)

Diferentemente das máquinas e computadores, que seguem proposições e comandos lógicos por meio de sistemas binários, os modelos mentais são conjuntos de modelos ou indicativos cognitivos que permitem analisar a veracidade de proposições em relação a modelos mentais de um estado de coisas no mundo.

Em suma, modelos mentais são construídos por indivíduos para representar estados físicos ou abstratos, a depender do fenômeno ou descritivo apresentado para análise. Por meio de interações com o sistema, os modelos mentais evoluem naturalmente no sentido de representar fielmente as funcionalidades que satisfaçam a correta interpretação do fenômeno, tendo como base as experiências anteriores e conhecimentos prévios do sujeito construtor ou "montador", utilizando o conceito de blocos cognitivos - do modelo mental.

Klimoski (1994) corrobora as definições propostas quando afirma que as estruturas dos modelos mentais são projetadas para auxiliar os processos interpretativos, possibilitando aos indivíduos o afastamento do conjunto total de informações e consequente prevenção do excesso de informações e níveis intoleráveis de incerteza.

Moreira (1996, p. 197 apud Norman 1996, p. 12), por sua vez, sugere que os modelos mentais têm as seguintes características gerais:

a) Modelos mentais são incompletos;

b) Existem claras e diversas limitações dos indivíduos em "rodar" seus modelos mentais;

c) Modelos mentais são instáveis e facilmente esquecíveis, principalmente quando não utilizados ou considerados por longos períodos de tempo;

d) Modelos mentais de dispositivos, operações e/ou fenômenos similares são frequentemente confundidos uns com os outros;

e) Indivíduos mantêm padrões de comportamento - e consequentes modelos mentais não científicos, ou seja, são incluídas tarefas supersticiosas ou vícios que não necessariamente alteram o resultado esperado do fenômeno;

f) Frequentemente as pessoas optam pelo aumento das tarefas físicas adicionais ao invés de maior comprometimento cognitivo, o que torna o modelo mental relacionado parcimonioso.

Os modelos mentais das pessoas podem incluir elementos desnecessários, errôneos, contraditórios ou ineficientes. Devem ser desenvolvidos, portanto, modelos estruturados de 
explicitação de conhecimentos que permitam aos indivíduos a formulação de modelos mentais adequados, mesmo que diferentes entre si.

\subsection{Comparação de Conceitos}

Considerando a nomenclatura semelhante, é possível inferir que os conceitos de Modelos Mentais (Mental Models) e Mapas da Mente (Mind Maps) seriam relacionados, embora esta inferência represente uma informação falsa.

Enquanto o conceito de Mapas Mentais refere-se a uma metodologia definida para análise de correlações entre causas e efeitos simulando o sequenciamento do pensamento humano, o conceito de Modelos Mentais refere-se à tentativa de representação das interações cognitivas e seus resultados na tentativa de representação de fenômenos físicos e/ou abstratos.

Considerando o exposto, serão construídos núcleos de pesquisa para os dois termos separadamente, uma vez que estes não apresentam sinergias teóricas.

\subsection{Perguntas de Pesquisa}

- Os termos “Mind Maps” e "Mental Models” são correspondentes ao mesmo conceito?

- Como os temas têm evoluído no tocante à representação na literatura?

\subsection{Objetivos}

Visando responder às perguntas de pesquisa, este artigo tem como objetivo à análise bibliográfica da evolução dos temas, assim como a comparação do conceito geral de ambos. Para a confecção das análises, os seguintes passos deverão ser seguidos:

- Apresentar uma breve discussão teórica sobre os termos;

- Construir uma base de publicações utilizando a Scopus;

- Analisar quantitativamente as publicações;

- Definir um conjunto de artigos para análise inicial e estruturação teórica dos conceitos (núcleo de pesquisa);

- Analisar e apresentar os resultados.

\section{Metodologia}

Esta seção apresentará a metodologia que será utilizada para a confecção deste artigo, visando permitir futuras análises e repetições do mesmo. Será utilizado o modelo interseccional entre 
a Bibliometria, o Blibliomining e a Webmetria proposto por Costa (2010), denominando Webibliomining. Conforme as proposições do autor, as etapas que compõem o modelo serão demonstradas a seguir, visando a melhor ilustração e facilitação do entendimento.

Figura 5 - Metodologia Proposta

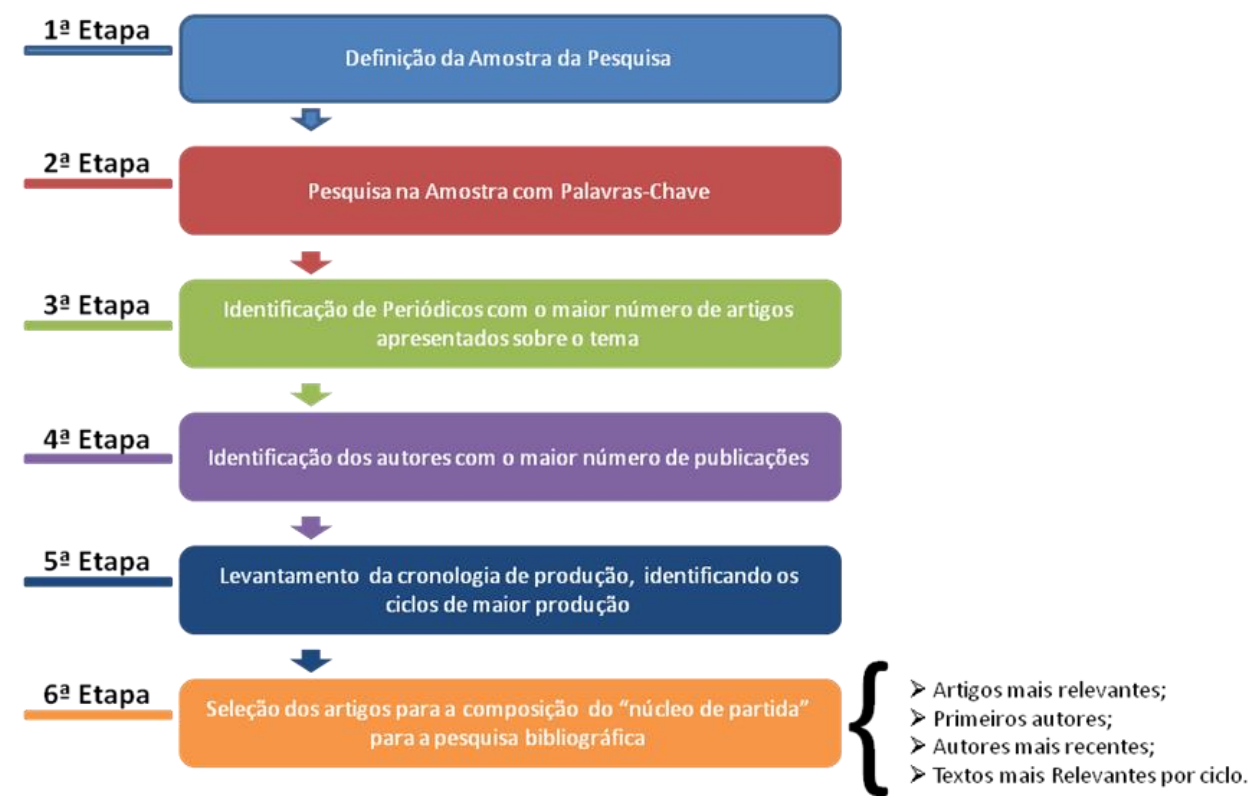

Fonte: Adaptado de Costa (2010, p. 116)

\section{Aplicação do Modelo}

Será descrita nesta seção a aplicação dos passos definidos no item anterior, visando obter as respostas às perguntas de pesquisa que este artigo se propõe e discutir.

\subsection{Definição da Amostra}

A amostra pesquisada corresponde aos materiais indexados na base de dados Scopus, com acesso por meio de registro individual no sistema da Universidade Federal Fluminense no mês de novembro de 2018. Considerando a extensão e abrangência da base de dados, esta foi utilizada como fonte única de análise e construção do banco de dados.

\subsection{Pesquisa na Amostra}

Conforme os temas centrais deste artigo, a busca foi efetuada utilizando-se os termos "Mind Maps" e "Mental Models" separadamente, podendo estes estar presentes no título, resumo ou 
palavras-chave dos materiais. A distribuição inicial dos achados considerando as áreas de pesquisa aos quais se relacionam será apresentada na Tabela 1.

Tabela 1 - Áreas de Concentração dos Trabalhos

\begin{tabular}{|c|c|c|}
\hline Subject Area & $\begin{array}{l}\text { Mind } \\
\text { Maps }\end{array}$ & $\begin{array}{l}\text { Mental } \\
\text { Models }\end{array}$ \\
\hline Computer Science & 286 & 2509 \\
\hline Social Sciences & 254 & 2369 \\
\hline Engineering & 134 & 1391 \\
\hline Medicine & 69 & 543 \\
\hline Mathematics & 51 & 650 \\
\hline Business, Management and Accounting & 48 & 911 \\
\hline Arts and Humanities & 38 & 810 \\
\hline Psychology & 37 & 1482 \\
\hline Decision Sciences & 26 & 263 \\
\hline Nursing & 26 & 94 \\
\hline Agricultural and Biological Sciences & 19 & 153 \\
\hline Environmental Science & 18 & 281 \\
\hline Health Professions & 13 & 83 \\
\hline Economics, Econometrics and Finance & 11 & 201 \\
\hline Physics and Astronomy & 10 & 104 \\
\hline Earth and Planetary Sciences & 7 & 97 \\
\hline Materials Science & 6 & 47 \\
\hline Chemistry & 5 & 51 \\
\hline Energy & 5 & 73 \\
\hline Multidisciplinary & 5 & 35 \\
\hline Biochemistry, Genetics and Molecular & 4 & 159 \\
\hline \multicolumn{3}{|l|}{ Biology } \\
\hline Chemical Engineering & 4 & 33 \\
\hline Dentistry & 3 & 2 \\
\hline Neuroscience & 3 & 324 \\
\hline Pharmacology, Toxicology and & 3 & 13 \\
\hline \multicolumn{3}{|l|}{ Pharmaceutics } \\
\hline Veterinary & 2 & 3 \\
\hline Undefined & 1 & 12 \\
\hline Total & 1088 & 12693 \\
\hline
\end{tabular}


Buscando aproximar a base de dados às respostas que este artigo se propõe a obter, foram selecionados somente os artigos com a temática "Business, Management and Accounting", sendo assim utilizados na base de dados 48 itens com a temática "Mind Maps" e 911 itens com a temática "Mental Models". A distribuição destes itens com base no tipo de documento será apresentada na Tabela 2.

Tabela 2 - Categorização por Tipo de Documento

\begin{tabular}{ccc}
\hline Tipo de Documento & Mind Maps & Mental Models \\
\hline Artigo & 33 & 697 \\
Conference Paper & 8 & 94 \\
Capítulo de Livro & 2 & 59 \\
Revisão & 1 & 36 \\
Artigo de Imprensa & 2 & 10 \\
Livro & 1 & 6 \\
Revisão para Conferência & 1 & 4 \\
Editorial & 0 & 2 \\
Nota & 0 & 2 \\
Errata & 0 & 1 \\
\hline
\end{tabular}

Fonte: Próprio Autor

Considerando o número de produtos encontrados, a pesquisa foi então refinada para que fossem considerados somente os artigos de periódicos. Assim sendo, a base de dados total será formada por 730 artigos, sendo 33 ligados à expressão-chave "Mind Maps" e 697 ligados à expressão "Mental Models".

Considerando a conclusão da escolha da base de dados foram então iniciadas as análises bibliométricas, cujos resultados serão apresentados e discutidos a seguir.

\subsection{Identificação dos periódicos com maior número de artigos publicados}

Foram encontradas publicações em 27 periódicos levando em consideração a temática "Mind Maps" e em 160 periódicos levando em consideração a temática "Mental Models". Os dez periódicos que mais publicaram artigos das temáticas "Mind Maps" e "Mental Models" serão apresentados nas Tabelas 3 e 4, respectivamente. Somente dez periódicos detêm publicações com os dois temas, e serão apresentados na Tabela 5. 
Tabela 3 - Periódicos que mais publicaram a respeito do tema "Mind Maps"

\begin{tabular}{lc}
\hline \multicolumn{1}{c}{ Título do Periódico } & Quantidade de Publicações \\
\hline Education Training & 2 \\
International Journal Of Consumer Studies & 2 \\
International Review Of Management And Marketing & 2 \\
Journal Of Management Development & 2 \\
Accounting Education & 1 \\
Annals Of Tourism Research & 1 \\
Career Development International & 1 \\
Education Business And Society Contemporary Middle Eastern & 1 \\
Issues & 1 \\
Espacios & 1 \\
Futures & 1 \\
\hline
\end{tabular}

Fonte: Próprio Autor

Tabela 4 - Periódicos que mais publicaram a respeito do tema "Mental Models"

\begin{tabular}{lr}
\hline \multicolumn{1}{c}{ Título do Periódico } & Quantidade de Publicações \\
\hline System Dynamics Review & 26 \\
Leadership Quarterly & 15 \\
Simulation And Gaming & 15 \\
Systems Research And Behavioral Science & 14 \\
Futures & 13 \\
Journal Of Risk Research & 13 \\
Learning Organization & 13 \\
Organizational Behavior And Human Decision Processes & 11 \\
Espacios & 10 \\
Strategic Management Journal & 10 \\
\hline
\end{tabular}


Tabela 5 - Periódicos que Publicaram nas temáticas de "Mind Maps" e "Mental Models"

\begin{tabular}{lrr}
\hline \multicolumn{1}{c}{ Titulo do Periódico } & Mind Maps & Mental Models \\
\hline Journal Of Management Development & 2 & 3 \\
Futures & 1 & 13 \\
Espacios & 1 & 10 \\
Technological Forecasting And Social Change & 1 & 9 \\
Annals Of Tourism Research & 1 & 3 \\
Knowledge And Process Management & 1 & 3 \\
Technovation & 1 & 3 \\
Industrial And Commercial Training & 1 & 2 \\
Technology In Society & 1 & 2 \\
Career Development International & 1 & 1 \\
\hline
\end{tabular}

Fonte: Próprio Autor

Mesmo considerando o baixo número de publicações com a temática de "Mind Maps", os periódicos listados na Tabela 5 são os que devem ser observados com mais atenção caso sejam feitos novos estudos visando identificar sinergias entre as temáticas.

\subsection{Identificação dos autores com maior número de publicações}

Considerando os recortes efetuados no banco de dados foi possível a confecção de uma listagem com os autores com o maior número de publicações nas duas temáticas, que será apresentado a seguir. O quadro foi elaborado considerando somente os autores com duas ou mais publicações na temática "Mind Maps" e quatro ou mais publicações da temática "Mental Models", visando efetuar um corte efetivo.

Tabela 6 - Autores com maior número de publicações na temática "Mind Maps"

\begin{tabular}{cc}
\hline Nome do Autor & Artigos \\
\hline Passerini, K. & 3 \\
Ilkevich, B.V. & 2 \\
Ilkevich, K.B. & 2 \\
Pollitt, D. & 2 \\
Pon, K. & 2 \\
Usmanova, L.T. & 2 \\
\hline
\end{tabular}


Tabela 7 - Autores com maior número de publicações na temática "Mental Models"

\begin{tabular}{cc}
\hline Nome do Autor & Artigos \\
\hline Mumford, M.D. & 11 \\
Hodgkinson, G.P. & 7 \\
Daniels, K. & 5 \\
Johnson, G. & 5 \\
Werhane, P.H. & 5 \\
Wood, R.E. & 5 \\
Woodside, A.G. & 5 \\
Gary, M.S. & 4 \\
Mohammed, S. & 4 \\
Passos, A.M. & 4 \\
Santos, C.M. & 4 \\
Sterman, J.D. & 4 \\
Strandvik, T. & 4 \\
Sushil & 4 \\
\hline
\end{tabular}

Fonte: Próprio Autor

Foi possível observar, considerando a totalidade do banco de dados de autores, que nenhum autor publicou nas duas temáticas até o momento.

\subsection{Levantamento das Cronologias de Produção}

Serão apresentados a seguir dois gráficos de horizonte temporal visando ilustrar a distribuição da confecção de artigos referentes aos dois temas. Enquanto o Gráfico 1 apresentará a evolução do tema "Mind Maps", o Gráfico 2 apresentará a evolução do tema "Mental Models". 
Gráfico 1 - Evolução das Publicações no Tema "Mind Maps"

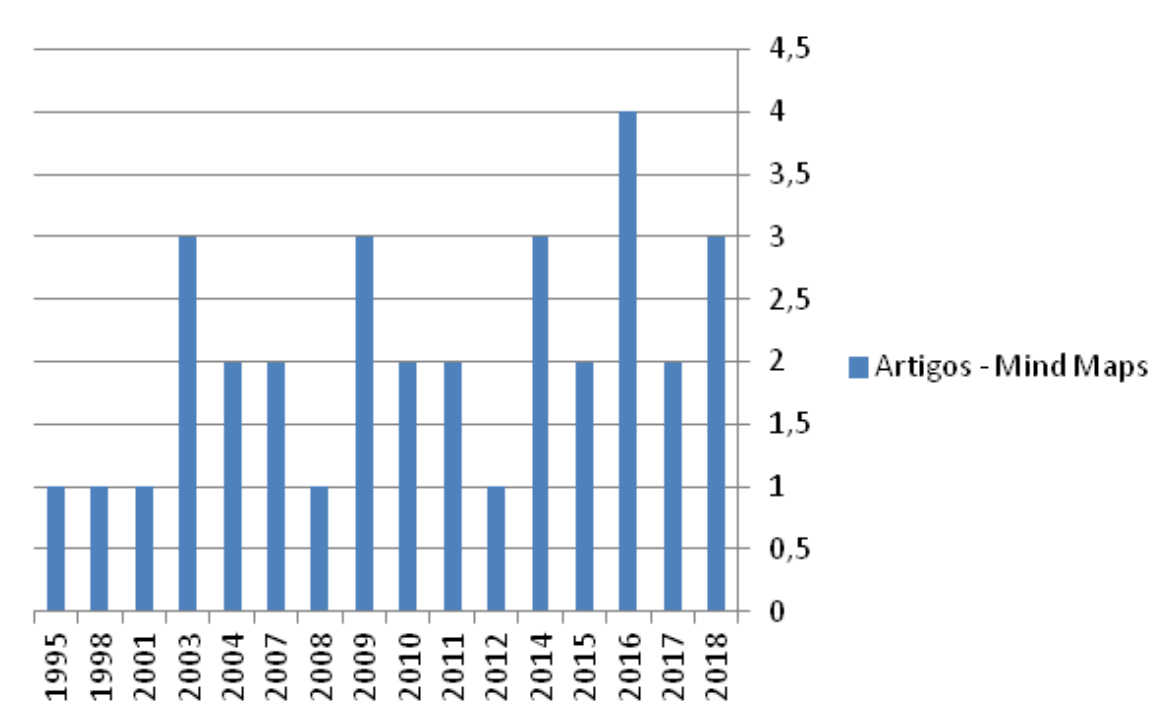

Fonte: Próprio Autor

Gráfico 2 - Evolução das Publicações no Tema "Mental Models"

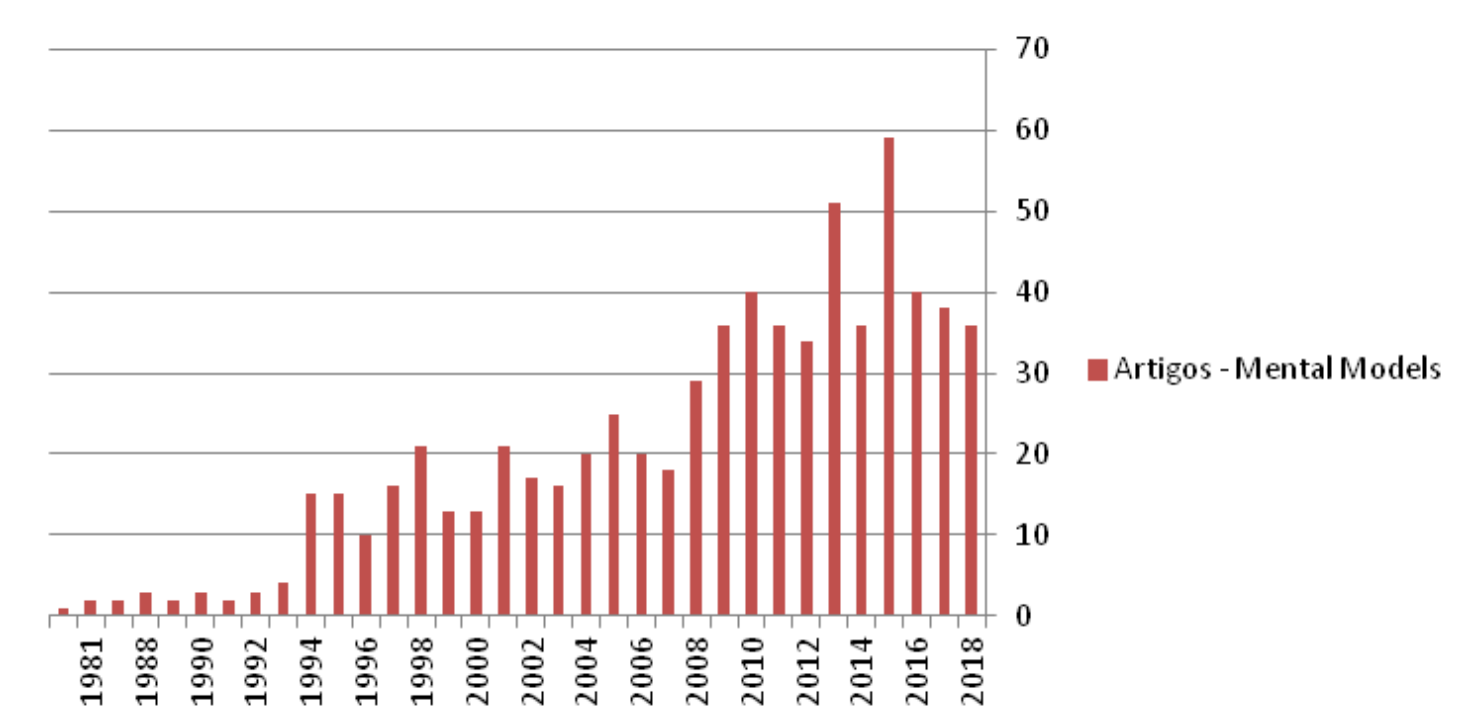

Fonte: Próprio Autor

Observando os gráficos é possível identificar uma tendência de crescimento referente à publicações ligadas à Mental Models até o ano de 2014, enquanto as publicações com a temática Mind Maps parecem manter-se no mesmo patamar quantitativo. 
Considerando os ciclos de publicação, serão considerados para construção do núcleo de pesquisa os seguintes períodos de produção acentuada:

- Mind Maps: 2016;

- Mental Models: 1994-1995,2004-2005, 2008-2010, 2013, 2015.

\subsection{Resultados Obtidos: Identificação do Núcleo de Partida}

A sexta etapa da metodologia proposta por Costa (2010) trata da seleção de um conjunto de artigos que servirão como referenciais iniciais para pesquisa bibliográfica referente aos temas propostos. Para tal, deverão ser utilizadas as seguintes regras:

a) Regra 1: Seleção dos três artigos (ou aproximadamente 1\% da base) mais antigos para identificação de linhas de pensamento diferentes nas discussões iniciais;

b) Regra 2: Visando dar mais ênfase aos artigos mais recentes em relação às discussões iniciais, devem ser selecionados os 15 artigos (ou aproximadamente 5\% da base) mais recentes de diferentes autores, com objetivo similar ao da primeira etapa;

c) Regra 3: Seleção dos 15 artigos (ou aproximadamente 5\% da base) com maior grau de relevância utilizando o algoritmo próprio da base de dados utilizada;

d) Regra 4: Seleção dos artigos com maior relevância em cada um dos períodos identificados na etapa anterior como de maior produção;

e) Regra 5: Montagem final do núcleo de partida por meio da integração de todos os itens selecionados nas etapas anteriores.

A seguir serão apresentados os resultados obtidos para cada uma das temáticas.

\subsubsection{Resultados Obtidos: Identificação do Núcleo de Partida da Temática Mind Maps}

Devido ao pequeno número de artigos disponíveis relacionados à temática, algumas observações devem ser feitas antes da apresentação do quadro final.

Nas regras 2 e 3, onde deveriam ser selecionados 15 artigos, foram selecionados 6. Isto se deve ao tamanho total da base de dados disponível e na busca da melhor adequação do núcleo de partida que será produto da análise. Como os artigos destacados na etapa 4 foram os mesmos identificados na etapa 3, a regra não foi considerada na construção da tabela final. A sumarização dos dados referente à regra 5 será apresentada na Tabela 7, que representará a totalidade do núcleo de partida desta temática. A Tabela 8, que será apresentada em seguida, representa o núcleo de partida da temática Mental Models. 
Tabela 8- Núcleo de Partida da Temática Mind Maps

\begin{tabular}{|c|c|c|c|c|}
\hline Etapa & Título & Autor & Ano & Periódico \\
\hline \multirow{6}{*}{$\begin{array}{c}\text { Regra } \\
1\end{array}$} & & & & Long Range \\
\hline & Henkel's strategy for Asia Pacific & Schütte, H. & 1995 & Planning 28 (1), pp. \\
\hline & & & & $95-103$ \\
\hline & Mind maps chart the way to & \multirow{2}{*}{ [No author name available] } & \multirow{2}{*}{1998} & Education + Training \\
\hline & business efficiency & & & 40(4), pp. 173-174 \\
\hline & $\begin{array}{l}\text { Transition in the making. The } \\
\text { images of the future in education } \\
\text { and decision-making }\end{array}$ & Rubin, A., Linturi, H. & 2001 & $\begin{array}{l}\text { Futures } 33(3-4), \mathrm{pp} . \\
\qquad 267-305\end{array}$ \\
\hline \multirow{25}{*}{$\begin{array}{c}\text { Regra } \\
2\end{array}$} & & \multirow{4}{*}{$\begin{array}{c}\text { Lopes, A.P.V.B.V., de Carvalho, } \\
\text { M.M. }\end{array}$} & \multirow{4}{*}{2018} & Technological \\
\hline & paradiom. Towards a contingent & & & Forecasting and \\
\hline & conceptual model & & & Social Change 132, \\
\hline & & & & pp. $284-298$ \\
\hline & Using Mind Maps to Distinguish & \multirow{4}{*}{$\begin{array}{l}\text { Bandera, C., Eminet, } \\
\text { A., Passerini, K., Pon, K. }\end{array}$} & \multirow{4}{*}{2018} & Journal of Small \\
\hline & Cultural Norms between French & & & Business \\
\hline & and United States & & & Management 56, pp. \\
\hline & Entrepreneurship Students & & & $177-196$ \\
\hline & Mind Mannino. An Fxneriential & \multirow{3}{*}{ McCrea, E.A., Lorenzet, S.J. } & \multirow{3}{*}{2018} & Organization \\
\hline & Approach to Syllabus Review & & & Management Journal \\
\hline & & & & $15(1)$, pp. $35-43$ \\
\hline & Factors in Agile methods & \multirow{2}{*}{ Kudryavtsev, D., Gavrilova, T. } & \multirow{2}{*}{2017} & TEM Journal 6 (2) \\
\hline & adoption & & & pp. $416-421$ \\
\hline & From Anarchy to System: A & \multirow{4}{*}{ Mikušová, M., Čopíková, A. } & \multirow{4}{*}{2017} & Knowledoe and \\
\hline & Novel Classification of Visual & & & Process Management \\
\hline & Knowledge Codification & & & \\
\hline & Techniques & & & \\
\hline & What Business Owners Expect & \multirow{4}{*}{$\begin{array}{c}\text { De Oliveira, F.R., Braga França, } \\
\text { S.L., De Souza, R.G., Duncan } \\
\text { Rangel, L.A. }\end{array}$} & \multirow{4}{*}{2016} & Journal of \\
\hline & From a Crisis Manager? A & & & Contingencies and \\
\hline & Competency Model: Survey & & & Crisis Management \\
\hline & Results From Czech Businesses & & & $24(3)$, pp. $162-180$ \\
\hline & Using mind maps to study how & & & International Journal \\
\hline & business school students & Somers, M.J., Passerini, & 2014 & of Management \\
\hline & andfaculty organize and apply & K., Parhankangas, A., Casal, J. & & Education 12(1), pp. \\
\hline & general business knowledge & & & $1-13$ \\
\hline \multirow{6}{*}{$\begin{array}{c}\text { Regra } \\
3\end{array}$} & & \multirow{6}{*}{$\begin{array}{c}\text { Hegazy, T., Ali, A., Abdel- } \\
\text { Monem, M. }\end{array}$} & \multirow{6}{*}{2011} & Journal of \\
\hline & & & & Professional Issues in \\
\hline & 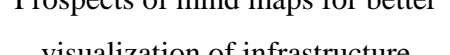 & & & Engineering \\
\hline & & & & Education and \\
\hline & & & & Practice 137(4), pp. \\
\hline & & & & $239-247$ \\
\hline
\end{tabular}




\subsubsection{Resultados Obtidos: Identificação do Núcleo de Partida da Temática Mental}

\section{Models}

Tabela 9 - Núcleo de Partida da Temática Mental Models

\begin{tabular}{|c|c|c|c|c|}
\hline Etapa & Título & Autor & Ano & Periódico \\
\hline \multirow[t]{11}{*}{ Regra 1} & An application of catastrophe & Graham, R., Seltzer, J. & 1979 & Omega 7(1), pp. 61- \\
\hline & theory to management science & & & 66 \\
\hline & process & & & \\
\hline & The function of mental imagery & Strizenec, M. & 1981 & Travail Humain \\
\hline & in work | [LA FONCTION DES & & & $44(2)$, pp. $283-288$ \\
\hline & REPRESENTATIONS & & & \\
\hline & MENTALES DANS LE & & & \\
\hline & TRAVAIL] & & & \\
\hline & Control bureaucracy and power & Brix, V.H. & 1981 & Human Systems \\
\hline & (a systems and cybernetic & & & Management 2(4), pp. \\
\hline & model) & & & $316-321$ \\
\hline \multirow[t]{28}{*}{ Regra 2} & Understanding team & Frick S.E., Fletcher K.A., & 2018 & Human Resource \\
\hline & maladaptation through the lens & Ramsay P.S., Bedwell W.L., & & Management Review \\
\hline & of the four R's of adaptation & & & $28(4)$, pp. $411-422$ \\
\hline & Creating a shared narrative: the & Ketonen-Oksi S., & 2018 & European Journal of \\
\hline & use of causal layered analysis to & & & Futures Research \\
\hline & explore value co-creation in a & & & $6(1), 5$ \\
\hline & novel service ecosystem & & & \\
\hline & Fostering entrepreneurial & Cosenz F., Noto G., & 2018 & International Journal \\
\hline & learning processes through & & & of Management \\
\hline & Dynamic Start-up business & & & Education 16(3), pp. \\
\hline & model simulators & & & $468-482$ \\
\hline & Visitor perspectives on & Winter M., & 2018 & Museum \\
\hline & commenting in museums & & & Management and \\
\hline & & & & Curatorship 33(5), pp. \\
\hline & & & & 484-505 \\
\hline & Sensemaking and sensegiving: & Will M.G., Pies I., & 2018 & Journal of Accounting \\
\hline & A concept for successful change & & & and Organizational \\
\hline & management that brings & & & Change \\
\hline & together moral foundations & & & 14(3), pp. 291-313 \\
\hline & theory and the ordonomic & & & \\
\hline & approach & & & \\
\hline & Inside-out and outside-in mental & Yrjölä M., Kuusela H., Neilimo & 2018 & European Business \\
\hline & models: a top executive & K., Saarijärvi H., & & Review 30(5), pp. \\
\hline & perspective & & & $529-553$ \\
\hline & Management accounting & Fletcher N.J., Ridley-Duff R.J., & 2018 & Qualitative Research \\
\hline & information and the board & & & in Accounting and \\
\hline & meeting of an English further & & & Management 15(3), \\
\hline & education college & & & pp. 313-340 \\
\hline
\end{tabular}

Fonte: Próprio Autor 


\section{Considerações Finais}

O trabalho desenvolvido obteve sucesso ao responder as perguntas de pesquisa às quais se propôs. Valendo-se de um modelo referencial breve, foi possível identificar que os conceitos de Mind Models e Mental Maps não tem significação semelhante, mesmo considerando que os termos no idioma português sugiram similaridade conceitual.

Considerando a diferença de significação foram construídos separadamente núcleos de pesquisa iniciais para cada uma das temáticas, utilizando a metodologia de Webibliomining. Por meio desta, foi possível ainda a identificação de informações quantitativas sobre a produção intelectual de cada uma delas, a saber:

- Artigos mais antigos;

- Artigos mais recentes;

- Artigos mais relevantes;

- Principais ciclos de produção textual;

- Artigos mais relevantes de cada ciclo;

- Principais autores;

- Principais periódicos.

Vale ressaltar que, embora a pesquisa tenha apresentado resultados satisfatórios, só foi consultada a base de dados Scopus, sendo ainda os artigos filtrados segundo a temática escolhida. Embora estas ações metodológicas auxiliem o direcionamento ao alcance dos objetivos do trabalho, representam também limitações que devem ser destacadas e podem ser superadas em trabalhos futuros a respeito do tema.

\section{REFERÊNCIAS}

COSTA, H. G. Modelo para webibliomining: proposta e caso de aplicação. Rev. FAE, Curitiba, v.13, n.1, p.115126, jan./jun. 2010.

DANIEL, P.A.; DANIEL, C. Complexity, uncertainty and mental models: From a paradigm of regulation to a paradigm of emergence in project management. International Journal of Project Management, v. 36, n. 1, p. 184-197, 2018.

EPPLER, M.J. A comparison between concept maps, mind maps, conceptual diagrams, and visual metaphors as complementary tools for knowledge construction and sharing. Information Visualization, v. 5, n. 3, p. 202-210, 2006.

GARY, M. S.; WOOD, R.E. Mental Models, Decision Rules and Performance Heterogeneity. Strat. Mgmt. J., v. 32, p. 569-594, 2011.

KLIMOSKI, R.; MOHAMMED, S. Team Mental Model: Construct or Metaphor?. Journal of Management, v. 20, n. 2, p. 403-437, 1994. 
MOREIRA, M. A. Modelos Mentais. Investigações em Ensino de Ciências, UFRGS, v. 1, n. 3, p. $193-232$. 1996.

SOMERS, M.J.; PASSERINI, K.; PARHANKANGAS, A.; CASAL, J. Using mind maps to study how business school students and faculty organize and apply general business knowledge. The International Journal of Management Education, v. 12, n. 1, p. 1-13, 2014.

YOAP, T. Fishbone Diagrams vs. Mind Maps. In: Tang, L.C., Goh, T.N., Yam, H.S., Yoap, T. Six Sigma: Advanced Tools for Black Belts and Master Black Belts, 2006, cap. 7, p. 85-91. 